\title{
A novel IncRNA, LUADT1, promotes lung adenocarcinoma proliferation via the epigenetic suppression of p27
}

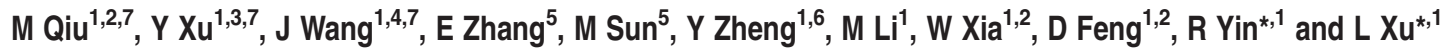

Long noncoding RNAs (IncRNAs) are known to regulate the development and progression of various cancers. However, few IncRNAs have been well characterized in lung adenocarcinoma (LUAD). Here, we identified the expression profile of IncRNAs and protein-coding genes via microarrays analysis of paired LUAD tissues and adjacent non-tumor tissues from five female nonsmokes with LUAD. A total of 498 IncRNAs and 1691 protein-coding genes were differentially expressed between LUAD tissues and paired adjacent normal tissues. A novel IncRNA, LUAD transcript 1 (LUADT1), which is highly expressed in LUAD and correlates with T stage, was characterized. Both in vitro and in vivo data showed that LUADT1 knockdown significantly inhibited proliferation of LUAD cells and induced cell cycle arrest at the GO-G1 phase. Further analysis indicated that LUADT1 may regulate cell cycle progression by epigenetically inhibiting the expression of p27. RNA immunoprecipitation and chromatin immunoprecipitation assays confirmed that LUADT1 binds to SUZ12, a core component of polycomb repressive complex 2, and mediates the trimethylation of $\mathrm{H} 3 \mathrm{~K} 27$ at the promoter region of p27. The negative correlation between LUADT1 and p27 expression was confirmed in LUAD tissue samples. These data suggested that a set of IncRNAs and protein-coding genes were differentially expressed in LUAD. LUADT1 is an oncogenic IncRNA that regulates LUAD progression, suggesting that dysregulated IncRNAs may serve as key regulatory factors in LUAD progression.

Cell Death and Disease (2015) 6, e1858; doi:10.1038/cddis.2015.203; published online 20 August 2015

Owing to smoking, air pollution and the aging of the population, the incidence and mortality rate of lung cancer is increasing rapidly. ${ }^{1}$ There is an obvious trend in recent years that the incidence of lung cancer caused by smoking is decreasing but that the incidence of lung adenocarcinoma (LUAD) in never smokers is growing. ${ }^{2,3}$ According recent statistical data, the percentage of non-smoker lung cancer is approximately $25 \%$ of all cases of lung cancer, including $15 \%$ of all male and $53 \%$ of all female lung cancer patients. It has been well documented that LUAD in never smokers is remarkably different from that in smokers with aspect to etiology, clinical characteristics, genomic and transcriptomic factors ${ }^{4}$ It is of paramount importance to identify the relationships between clinical symptoms and the molecular changes of LUAD among never smokers to develop new diagnostic and treatment strategies for LUAD and to improve the prognosis of diagnosed patients.

Long noncoding RNA (IncRNA) is a type of RNA molecules larger than 200 nucleotides that lacks protein-coding capacity. ${ }^{5,6}$ Owing to their lack of reading frames, IncRNAs were originally considered as transcriptional noise. However, emerging evidence has demonstrated that IncRNAs have important roles in various biological and pathological processes, such as the immune response, ${ }^{7}$ differentiation, ${ }^{8}$ metabolism, ${ }^{9}$ and cancer development and progression. ${ }^{10-12}$ As an emerging paradigm of cancer research, many cancerspecific IncRNAs have been identified, a set of which have been validated as biomarkers for metastasis or prognosis, such as metastasis associated long antisense transcript 1 (MALAT-1), ${ }^{13}$ HOX transcript antisense RNA (HOTAIR) ${ }^{14}$ and colon cancer-associated transcript 2 (CCAT2). MALAT-1, as indicated by its name, is a IncRNA that is highly expressed in metastatic LUAD and associated with poor prognosis. ${ }^{13,15}$ Currently, high-throughput technology such as RNA-sequencing and microarrays analysis has enable the characterization of IncRNA expression profile in biological processes ${ }^{16-18}$ and diseases. ${ }^{19-21}$

We have focused on IncRNA and reported a LUAD-specific IncRNA, CCAT2 that is significantly upregulated in LUAD but not in lung squamous cell cancer (LSCC). ${ }^{22}$ Here, we reported

\footnotetext{
${ }^{1}$ Department of Thoracic Surgery, Nanjing Medical University Affiliated Cancer Hospital, Jiangsu Key Laboratory of Molecular and Translational Cancer Research, Cancer Institute of Jiangsu Province, Baiziting 42, Nanjing 210009, China; ${ }^{2}$ The Fourth Clinical College of Nanjing Medical University, Nanjing, 210000, China; ${ }^{3}$ The First Clinical College of Nanjing Medical University, Nanjing 210000, China; ${ }^{4}$ Department of Scientific Research, Nanjing Medical University Affiliated Cancer Hospital, Cancer Institute of Jiangsu Province, Nanjing 210009, China; ${ }^{5}$ Department of Biochemistry and Molecular Biology, Nanjing Medical University, Nanjing 210000 , China and ${ }^{6}$ Department of Nursing, Nanjing Medical University Affiliated Cancer Hospital, Cancer Institute of Jiangsu Province, Nanjing 210009, China

${ }^{*}$ Corresponding author: R Yin or L Xu, Department of Thoracic Surgery, Nanjing Medical University Affiliated Cancer Hospital, Jiangsu Key Laboratory of Molecular and Translational Cancer Research, Cancer Institute of Jiangsu Province, Baiziting 42, Nanjing, 210009, China. Tel: +86 2583284700 ; Fax: +86 2583641062 ; E-mail: yinhero001@126.com or xulin83cn@gmail.com

${ }^{7}$ These authors contributed equally to this work.

Abbreviations: LUAD, lung adenocarcinoma; RIP, RNA immunoprecipitation; H3K27, histone 3 lysine 27; ChIP, chromatin immunoprecipitation; LUADT1, lung adenocarcinoma transcript 1; PRC2, polycomb repressive complex 2

Received 13.3.2015; revised 17.6.2015; accepted 24.6.2015; Edited by E Candi
} 
the protein-coding genes and IncRNAs expression profile of LUAD in female non-smokers characterized by microarrays and the identification of a novel IncRNA LUAD transcript 1 (LUADT1). The LUADT1 gene is located at chromosomal locus $6 q 24.3$ and transcribes a 453nt transcript. By binding to SUZ12, a core component of the polycomb repressive complex 2 (PRC2), LUADT1 epigenetically suppressed p27 expression via histone modification. The silence of LUADT1 induced cell cycle arrest and significantly inhibited tumor growth both in vivo and in vitro.

\section{Results}

Expression profiles of IncRNAs and protein-coding genes among never smokers with LUAD. We used microarray analysis to characterize the expression profiles of IncRNAs and protein-coding genes in five never-smoking female LUAD patients. As shown in Figure 1a, most IncRNAs analyzed in the expression profile have not been annotated (Figure 1a). Filtered by $P$-value and fold change $(P<0.05$ and fold change $>2.5$ for IncRNAs; $P<0.05$ and fold change $>3$ for protein-coding genes), a total of 498 IncRNAs and 1691 protein-coding genes showed differential expression between the LUAD and paired adjacent normal tissues (Figures $1 \mathrm{~b}$ and $\mathrm{c}$ ). To validate microarray findings, five differentially expressed IncRNAs were selected and analyzed in a cohort of 20 female LUAD patients without smoking history (Supplementary Table S3) using qRT-PCR (Figure 1d). In agreement with microarray results, AFAP1-AS1, PIK3CDAS2 and AC093850.2 were overexpressed and TINCR and TARID were downregulated in LUAD tissues $(P<0.05$ for all five IncRNAs). The top 100 differentially expressed IncRNAs and protein-coding genes are provided in Supplementary Table S4 and the microarray data have been uploaded to Gene Expression Omnibus database (accession number: GSE66654).

Transcription factors (TFs) have a central role in the regulation of gene expression. We constructed a TF-IncRNA-protein-coding gene network to illustrate how TFs modulate gene expression to identify the powerful TFs in LUAD. $^{23,24}$ As shown in Figure 1e, CPBP, ZNF333 and NF-AT1 were the three most dominant TFs, which affected the transcription of $>140$ genes. In addition, HIF1-alpha, SMAD4 and other oncogenic TFs also affect many genes. The co-expression networks were significantly different between the LUAD and normal lung tissues, suggesting that IncRNAs and protein-coding genes displayed different co-expression patterns between LUAD and normal tissues (Supplementary Figures S5 and S6). Gene ontology and KEGG pathway analyses were performed to identify the aberrant cellular functions and pathways (Supplementary Tables S7 and S8).

The novel IncRNA LUADT1 is upregulated in LUAD. Using hierarchical clustering, ${ }^{25,26}$ a widely used data-mining tool, we identified a set of significantly differentially expressed IncRNAs (Figure 2a). As co-expression modules can represent biological pathways, ${ }^{27}$ we next analyzed subnetworks of these IncRNAs and their related protein-coding genes. Using this approach, ${ }^{28}$ we characterized a novel
IncRNA, LUADT1, because its network included multiple protein-coding genes associated with tumor growth, invasion or prognosis (Figure 2b). The LUADT1 (ENSG00000196634) gene is located at chromosomal locus 6q24.3 locus (Figure 2c), which is within the lung cancer susceptibility locus $6 q 23-6 q 25,{ }^{29,30}$ and is transcribed as a 453nt IncRNA. The negative score of PhyloCSF ${ }^{31}(-12.7273$, meaning that LUADT1 is $10^{1.27273}$ times more likely to be a noncoding sequence than a coding sequence), a comparative genomic method differentiating coding and noncoding RNA, and the lack of coding potential as determined by the coding potential assessment tool CPAT $^{32}$ confirmed that LUADT1 is a noncoding RNA. Quantitative real-time PCR (qRT-PCR) was utilized to profile LUADT1 expression in lung cancer cell lines (Figure 2d). Compared with human bronchial epithelium (HBE), LUADT1 was remarkably overexpressed in the A549 cell line. In another cohort of 78 lung cancer patients, we investigated the expression level of LUADT1 and analyzed its clinical correlation (Table 1). As shown, LUADT1 was significantly overexpressed in lung tumor tissues compared with paired adjacent normal lung tissues, displaying an average difference of 8.34-fold (Figure 2e). The LUADT1 expression level was significantly higher in LUAD than in LSCC ( $P=0.019$; Figure 2f) and correlated with the tumor stage $(P=0.043$; Figure $2 \mathrm{~g}$ ), but not with $\mathrm{N}$ stage or TNM stage.

LUADT1 promotes cell proliferation in vitro. To evaluate the effects of LUADT1 on cell biological behavior, small interfering RNAs (siRNAs) were designed to silence LUADT1. The qRT-PCR results revealed that LAUDT1 was sufficiently silenced by siRNAs in two LUAD cell lines, A549 and $\mathrm{H} 1975$ (Figure 3a). Compared with the negative control (NC) siRNA, siRNAs targeting LUADT1 significantly inhibited cell proliferation ability in LUAD cell lines based on the CCK-8 assay (Figure $3 b$ ). Consistent with the results of the CCK-8 assay, colony formation ability was inhibited after LUADT1 knockdown as demonstrated by the decrease in the size and the number of colonies after siRNA-LUADT1 treatment (Figure 3c) compared with the NC treatment. We next examined whether the tumor cell cycle was affected after LUADT1 knockdown by Annexin $\mathrm{V}$ and propidium iodide double staining via FACS analysis. The results revealed that siRNA-LUADT1 treatment induced significant G0-G1 phase arrest and decreased the percentage of cells in the $S$ phase (Figure 3d). BrdU assay also revealed that cell proliferation ability was significantly inhibited in both A549 and H1975 cells (Figure $3 e$ ). In consistence with BrdU and CCK-8 results, the expression of proliferation biomarkers, cyclin D1, cyclin-dependent kinase 4 (CDK4), and cyclin-dependent kinase 6 (CDK6) were decreased after LUADT1 silence (Figure 3f). Apoptosis was also analyzed after silence of LUADT1. Compared with NC, the rate of apoptotic cells was not affected by siRNA-LUADT1 treatment (Supplementary Figure S9). These data demonstrated that LUADT1 may promote LUAD cell proliferation, as well as cell cycle progression.

The silence of LUADT1 suppressed tumor growth in vivo. To test whether LUADT1 regulates LUAD cell 
a

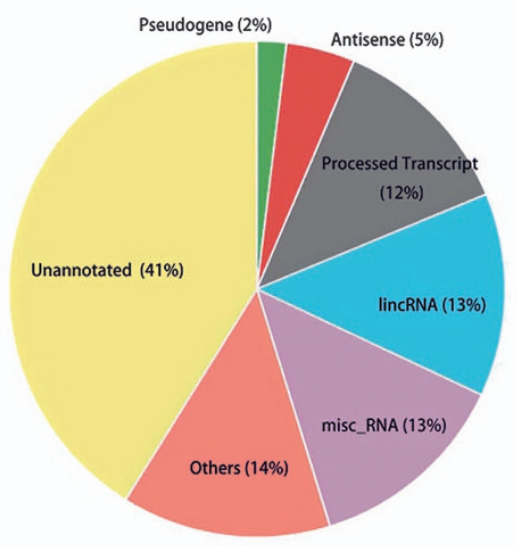

C
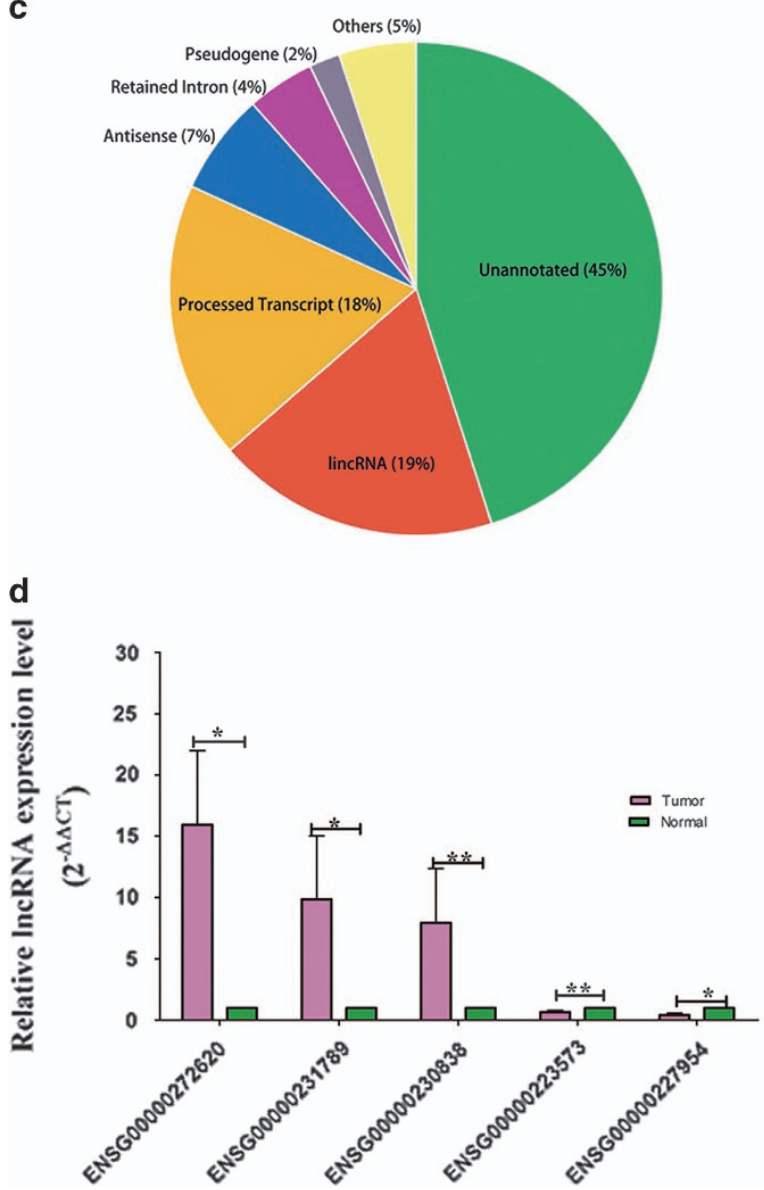

b

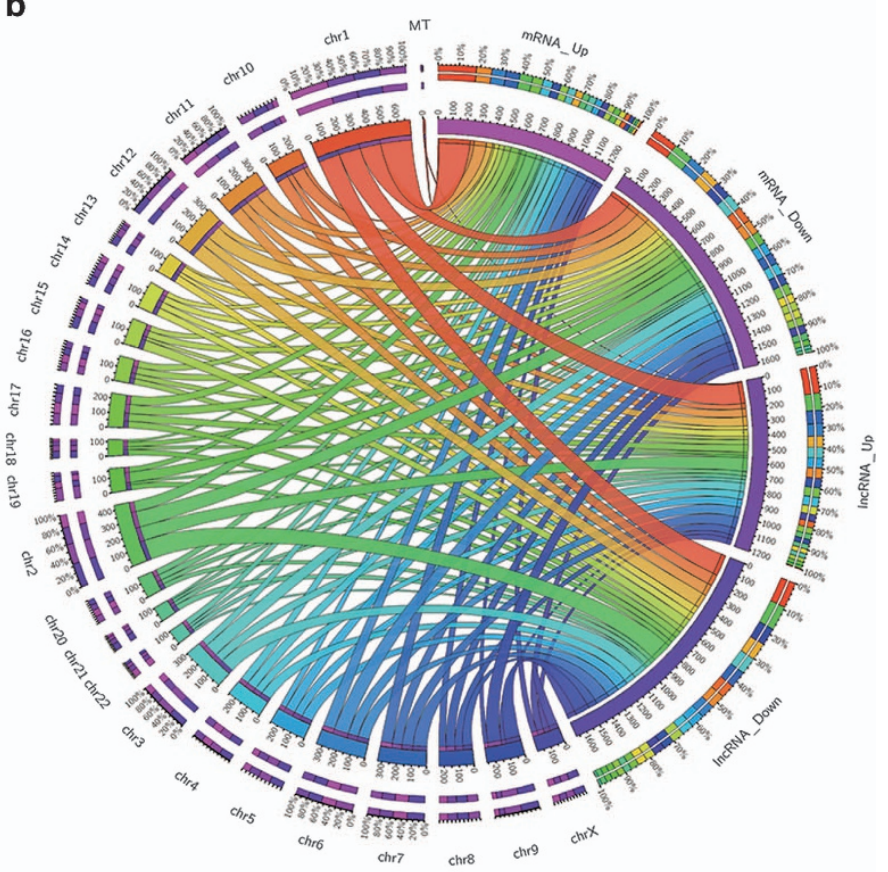

e

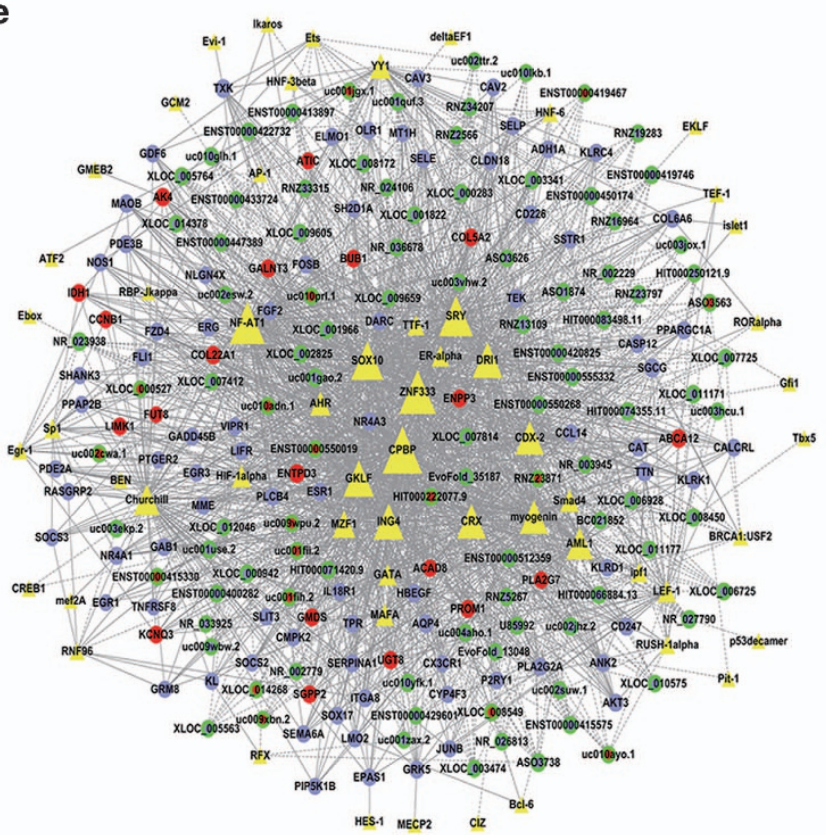

Figure 1 Expression profiling of IncRNAs and protein-coding genes via microarray analysis. (a) Biotypes of IncRNAs profiled. (b) Differentially expressed IncRNAs and protein-coding genes presented as a Circos plot. (c) Biotypes of differentially expressed IncRNAs. (d) The microarray results of five differentially expressed IncRNAs were selected for validation in 20 LUAD patients. ENSG00000272620: AFAP1-AS1; ENSG00000231789: PIK3CD-AS2; ENSG00000230838: AC093850.2; ENSG00000223573: TINCR; ENSG00000227954: TARID. ${ }^{*} P<0.05,{ }^{* \star} P<0.01$. (e) TF-IncRNA-protein-coding gene network; yellow: TFs; red: upregulated protein-coding genes; purple: downregulated protein-coding genes; green and red: upregulated IncRNAs; green and purple: downregulated IncRNAs. Error bars indicate means \pm S.E.M.

proliferation in vivo, we established xenograft tumor models in nude mice using A549 cells transfected with scrambled shRNA or shLUADT1. All nude mice developed xenograft tumors at the injection site, and the xenograft tumors were harvested 14 days after injection. qRT-PCR confirmed that the LUADT1 expression level was lower in the xenograft tumors derived from the shLUADT1-transfected cells (Figure 4a). As shown, the average tumor volume and weight in the shLUADT1 group was significantly lower than that in the control group (Figures $4 b-d$ ). IHC analysis revealed that tumors derived from shLUADT1-transfected cells showed weaker staining for Ki67, a cell proliferation marker, ${ }^{33}$ 
a

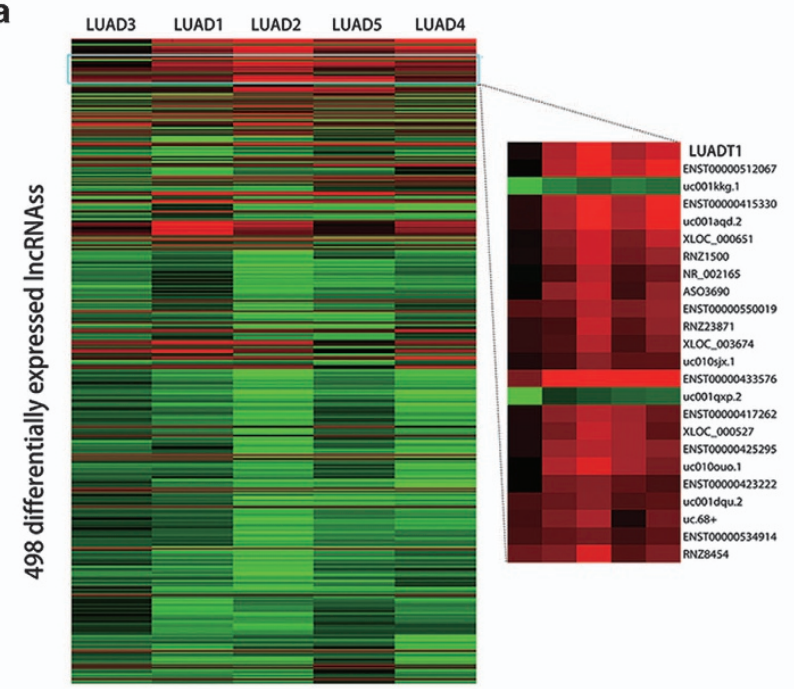

b

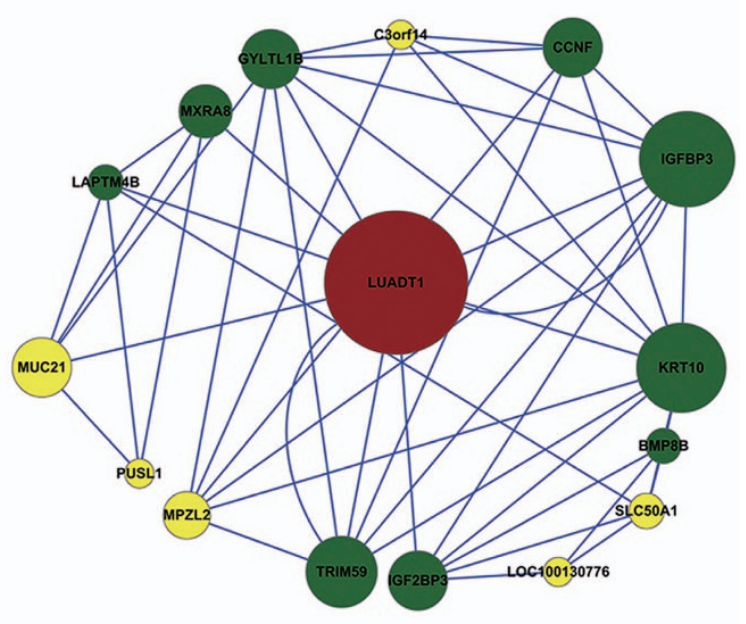

c

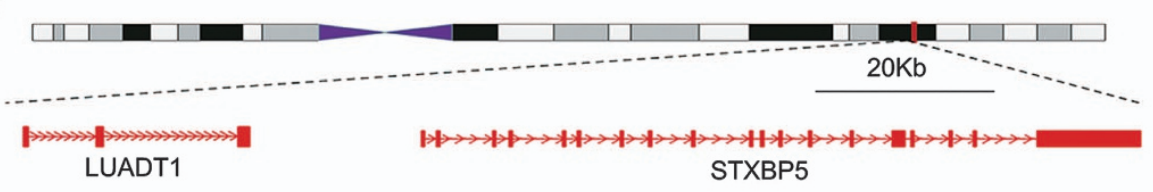

d

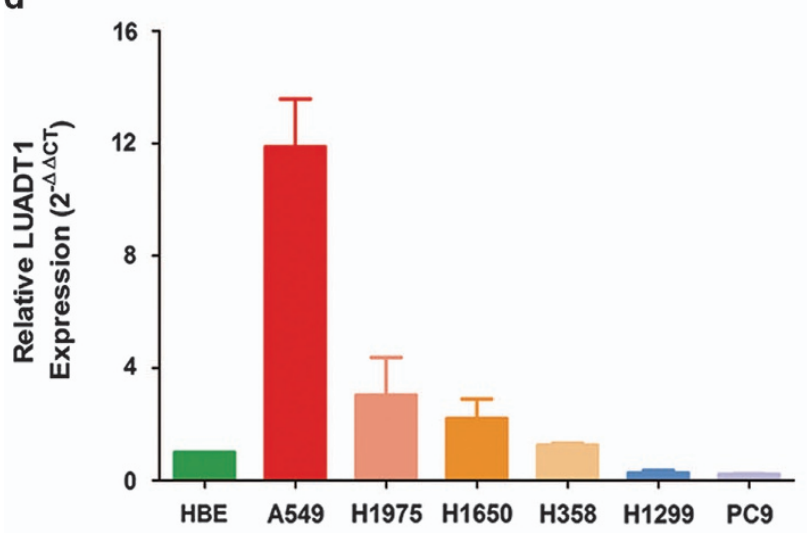

f

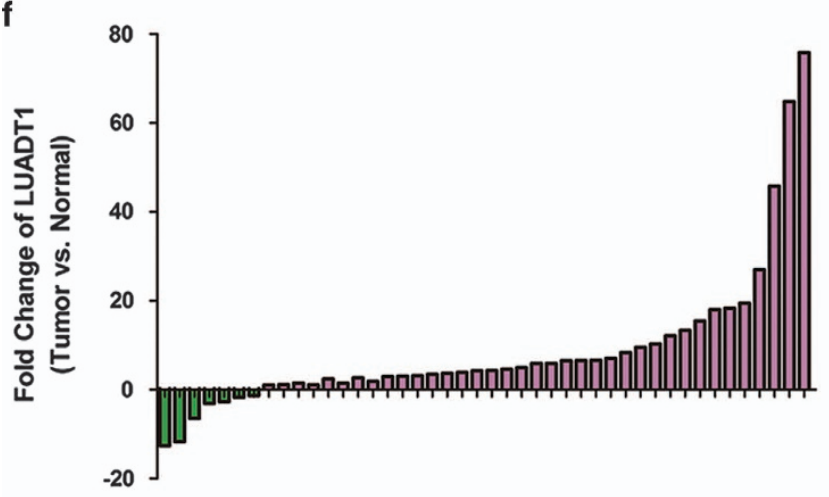

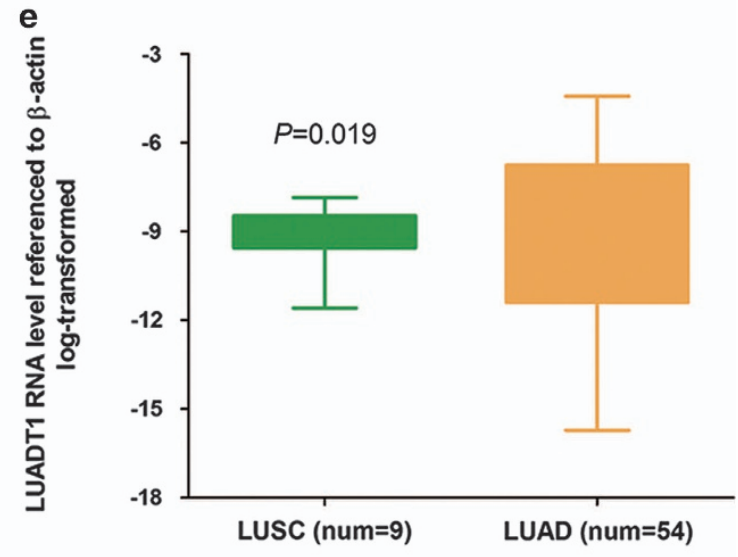

g

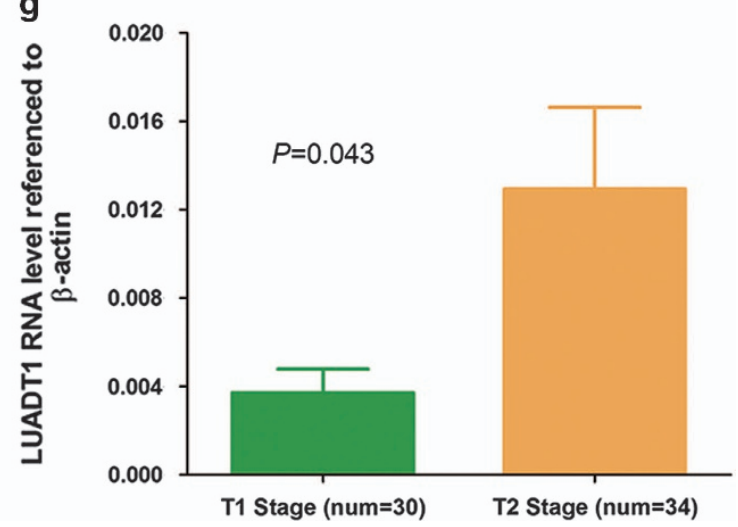

Figure 2 LUADT1 is upregulated in LUAD. (a) Hierarchical clustering showed that LUADT1 is significantly highly expressed; green: downregulated IncRNAs; red: upregulated IncRNAs. (b) Co-expression sub-network of LUADT1; green: genes associated with cell proliferation, drug resistance, tumor growth, invasion or prognosis; yellow: genes with unknown function. (c) Chromosomal location of LUADT1. (d) Expression of LUADT1 in LUAD cell lines. (e) LUADT1 is upregulated in NSCLC tissues compared with non-tumor tissues (8.34-fold, $P<0.05$ ). The LUADT1 expression level is higher in LUAD tissues than in LSCC tissues (f) and is higher in T2 stage tumors than in T1 stage tumors ( $\mathbf{g}$ ). Error bars indicate means \pm S.E.M. 
Table 1 Correlation between LUADT1 expression level and clinical features in lung adenocarcinoma

\begin{tabular}{|c|c|c|c|c|}
\hline Characteristics & $\begin{array}{c}\text { Number of } \\
\text { patients }\end{array}$ & Percentage & Expression ${ }^{a}$ & $P$-values \\
\hline \multicolumn{5}{|l|}{ Age (years) } \\
\hline $\begin{array}{l}<65 \\
>65\end{array}$ & $\begin{array}{l}42 \\
24\end{array}$ & $\begin{array}{l}63.64 \% \\
36.36 \%\end{array}$ & $\begin{array}{l}0.00848 \\
0.00827\end{array}$ & 0.961 \\
\hline \multicolumn{5}{|l|}{ Gender } \\
\hline Male & 33 & $50 \%$ & 0.01079 & \multirow{2}{*}{0.246} \\
\hline Female & 33 & $50 \%$ & 0.00603 & \\
\hline \multicolumn{5}{|l|}{ Smoking } \\
\hline Yes & 17 & $25.76 \%$ & 0.00653 & \multirow[t]{2}{*}{0.151} \\
\hline No & 49 & $74.24 \%$ & 0.01303 & \\
\hline \multicolumn{5}{|l|}{ Drinking } \\
\hline Yes & 6 & $9.09 \%$ & 0.01688 & \multirow[t]{2}{*}{0.191} \\
\hline No & 60 & $90.91 \%$ & 0.00756 & \\
\hline \multicolumn{5}{|l|}{ Family history } \\
\hline Yes & 7 & $10.61 \%$ & 0.00176 & \multirow[t]{2}{*}{0.265} \\
\hline No & 59 & $89.39 \%$ & 0.00920 & \\
\hline \multicolumn{5}{|l|}{ Histology type } \\
\hline SCC & 9 & $13.64 \%$ & 0.00249 & \multirow[t]{3}{*}{$0.037^{\mathrm{b}}$} \\
\hline$A D$ & 54 & $81.82 \%$ & 0.00751 & \\
\hline Others & 3 & $4.54 \%$ & 0.00286 & \\
\hline \multicolumn{5}{|l|}{ Tumor size } \\
\hline$<3 \mathrm{~cm}$ & 17 & $26.15 \%$ & 0.00421 & \multirow[t]{2}{*}{0.278} \\
\hline$>3 \mathrm{~cm}$ & 48 & $73.85 \%$ & 0.00823 & \\
\hline \multicolumn{5}{|l|}{ Differentiation } \\
\hline Low & 5 & $7.94 \%$ & 0.00120 & \multirow[t]{3}{*}{0.553} \\
\hline Middle & 40 & $63.49 \%$ & 0.00667 & \\
\hline High & 18 & $28.57 \%$ & 0.00804 & \\
\hline \multicolumn{5}{|l|}{$T$ stage } \\
\hline $\mathrm{T} 1$ & 30 & $46.86 \%$ & 0.00372 & \multirow[t]{2}{*}{$0.043^{b}$} \\
\hline $\mathrm{T} 2$ & 34 & $53.14 \%$ & 0.01036 & \\
\hline \multicolumn{5}{|c|}{ Lymph node metastasis } \\
\hline Yes & 21 & $31.82 \%$ & 0.006615 & \multirow[t]{2}{*}{0.845} \\
\hline No & 45 & $68.18 \%$ & 0.00729 & \\
\hline \multicolumn{5}{|l|}{ TNM stage } \\
\hline I & 39 & $59.09 \%$ & 0.00825 & \multirow[t]{4}{*}{0.774} \\
\hline II & 9 & $13.64 \%$ & 0.00449 & \\
\hline III & 16 & $24.24 \%$ & 0.00642 & \\
\hline IV & 2 & $3.03 \%$ & 0.00103 & \\
\hline
\end{tabular}

${ }^{\mathrm{a}}$ Expression level relative to $\beta$-actin. ${ }^{\mathrm{b}}$ Significant association

than those from scrambled shRNA-transfected cells (Figure $4 \mathrm{e}$ ). These data demonstrated that LUADT1 may regulate tumor cell growth in vivo.

LUADT1 suppresses p27 by binding to PRC2. As G0-G1 cell cycle arrest after LUADT1 knockdown was observed, we further explored the underlying mechanism using A549 as cell model. To identify the downstream targets of LUADT1, we first assessed the expression of the cyclin-dependent kinase inhibitor family via qRT-PCR (Figure 5a). Among these analyzed genes, we found that p27 (CDKN1B) was the most significantly upregulated gene after LUADT1 knockdown, and this result was confirmed by western blot (Figure 5b). p27 is an important tumor suppressor that is responsible for cell cycle control. ${ }^{34}$ Thus, we hypothesized that LUADT1 may promote LUAD cell proliferation via the suppression of p27 expression.

Subcellular location may provide clues regarding the molecular mechanism. Glyceraldehyde-3-phosphate dehydrogenase (GAPDH) and small nuclear RNA U1 (RNU1) were utilized as control of cytoplasm and nucleus, respectively. Compared with GAPDH and RNU1, LUADT1 was predominantly located in nucleus (Figure $5 \mathrm{c}$ ). In addition, to visualize LUADT1 expression and subcellular location, fluorescence in situ hybridization (FISH) assay was performed. As shown (Figure 5d), most LUADT1 was localized in nucleus in both A549 and H1975. Khalil et al. ${ }^{35}$ have reported that approximately $20 \%$ of IncRNAs bind to PRC2, indicating that most IncRNAs exert their biological function by binding to RNAbinding proteins (RBPs), especially PRC2. Thus, we hypothesized that LUADT1 may regulate $\mathrm{p} 27$ expression by recruiting PRC2. PRC2 consists of three components, EZH2, SUZ12 and EED1. We first predicted the binding ability of LUADT1 to two key components, EZH2 and SUZ12, using an online algorithm, RPISeq. ${ }^{36}$ Using the EZH2-HOTAIR interaction pair as a positive control (interaction probability $=0.75$ ), we found that the SUZ12-LUADT1 interaction pair (interaction probability $=0.7$ ) had a higher score than the EZH2-LUADT1 interaction pair (interaction probability $=0.45$ ). These results were confirmed using another online tool, catRAPID. ${ }^{37}$ Next, we performed RNA immunoprecipitation (RIP) using antibodies against SUZ12 and EZH2 and observed a significant enrichment of LUADT1 using the SUZ12 antibody, but not the $\mathrm{EZH} 2$ antibody (Figure 5e), compared with the nonspecific IgG control antibody. Together, these data confirmed physical interaction between SUZ12 and LUADT1.

We further investigated the functional relevance of the interaction between SUZ12 and LUADT1. SUZ12 was first silenced using siRNA, and significant upregulation of p27 was observed (Figure 5f). Current evidence has demonstrated that the PRC2 complex is a negative regulator of transcription via histone modification, that is, the trimethylation of histone 3 lysine 27 (H3K27me3). ${ }^{38}$ Thus, it is very likely that LUADT1 suppresses p27 expression by recruiting the PRC2 complex to p27 promoter region, leading to trimethylation of $\mathrm{H} 3 \mathrm{~K} 27$ at this region. By performing chromatin immunoprecipitation (ChIP) experiments using antibodies against SUZ12 and H3K27me3, we detected the enrichment of SUZ12 and H3K27me3 in the promoter region of p27 (Figure $5 \mathrm{~g}$ ). After LUADT1 silencing using siRNA, SUZ12 enrichment in the promoter region of p27 was significantly decreased, and the occupancy of H3K27me3 in the p27 locus also decreased (Figure 5h).

Based on the above findings, we examined whether LUADT1 regulates p27 in lung cancer patients. For this purpose, we first analyzed our microarray results and found that the expression levels of p27 and LUADT1 were negatively correlated. Next, in a published microarray data series of 117 LUAD patients (GSE37138), ${ }^{39}$ we confirmed that the expression level of p27 negatively correlated with that of LUADT1 (Figure 6a). In the expression cohort of 20 LUAD patients, qRT-PCR results showed that the p27 mRNA level negatively correlated with the expression of LUADT1 (Figure 6b). Consistent with in these results, IHC analysis revealed that 

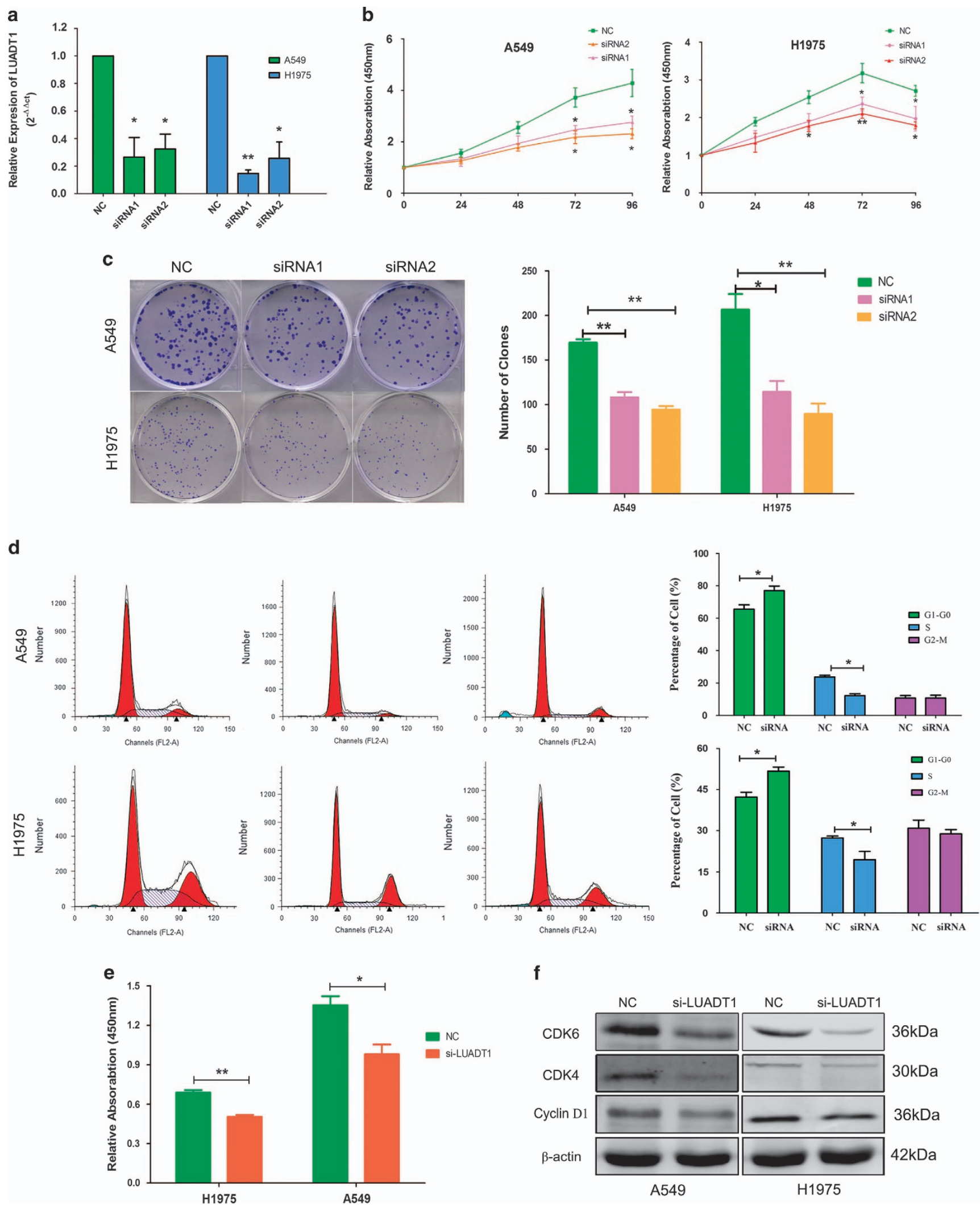

f

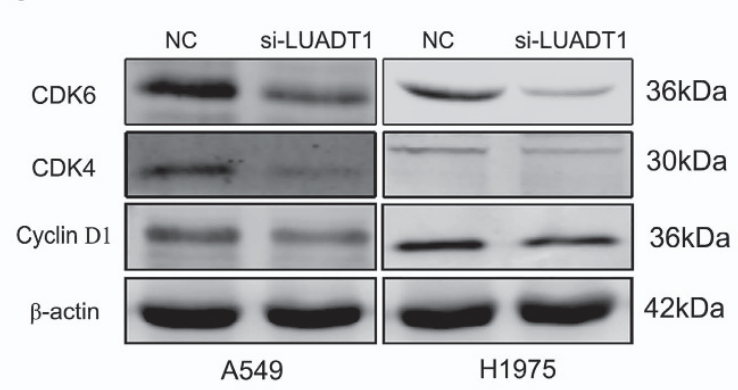

Figure 3 The silencing of LUADT1 inhibited LUAD cell proliferation in vitro. (a) Targeted siRNAs efficiently silenced LUADT1 in the A549 and H1975 cell lines. LUADT1 knockdown inhibited cell proliferation (CCK-8 assay, b) and clone formation (c) ability and induced cell cycle arrest at the G0-G1 stage (d) in A549 and H1975 cells. BrdU assay showed that siRNA-LUADT1 treatment significantly decreased relative absorbance at $450 \mathrm{~nm}$ (e). Western blot showed that cyclin D1, CDK4 and CDK6 were decreased after LUADT1 silence in A549 and H1975 cells (f). ${ }^{*} P<0.05,{ }^{* *} P<0.01$. Error bars indicate means \pm S.E.M. 

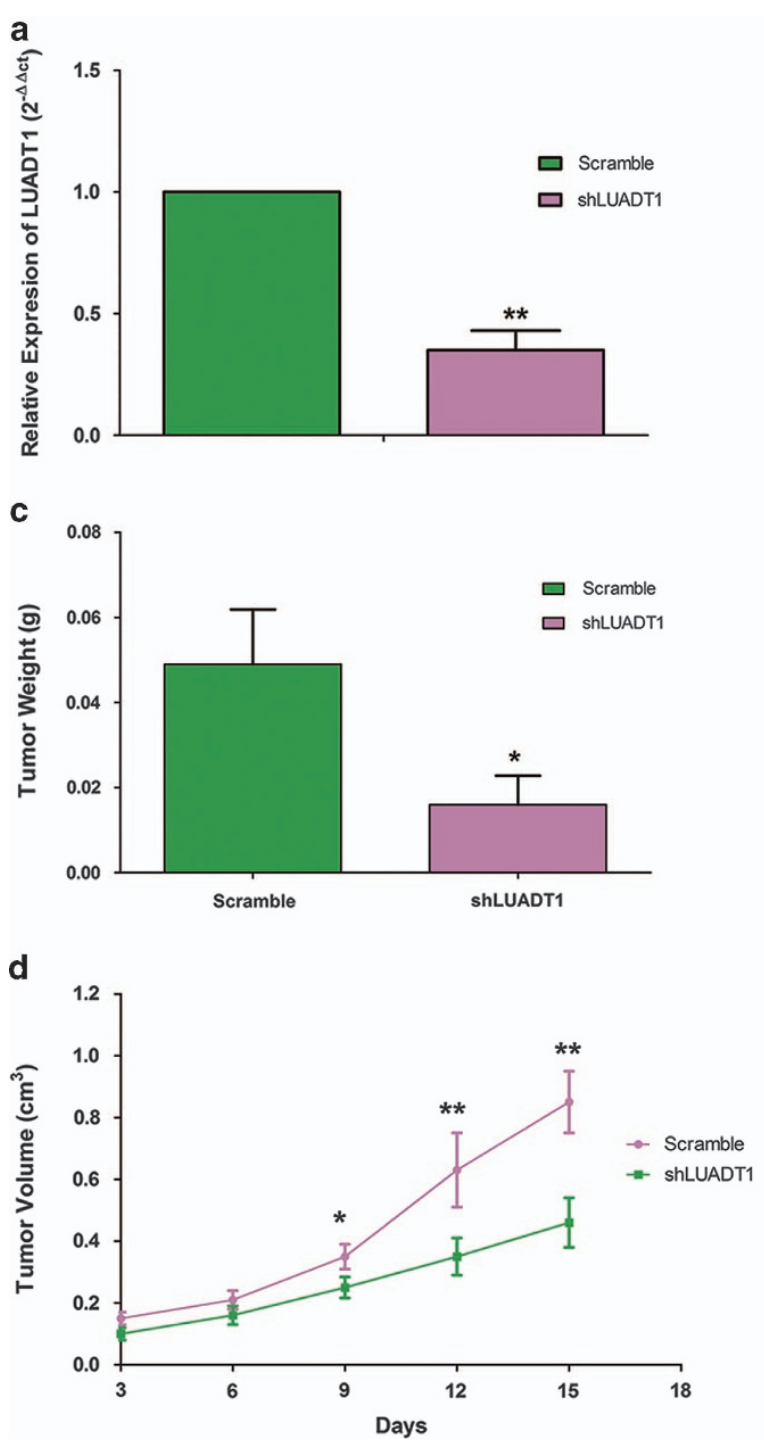

b

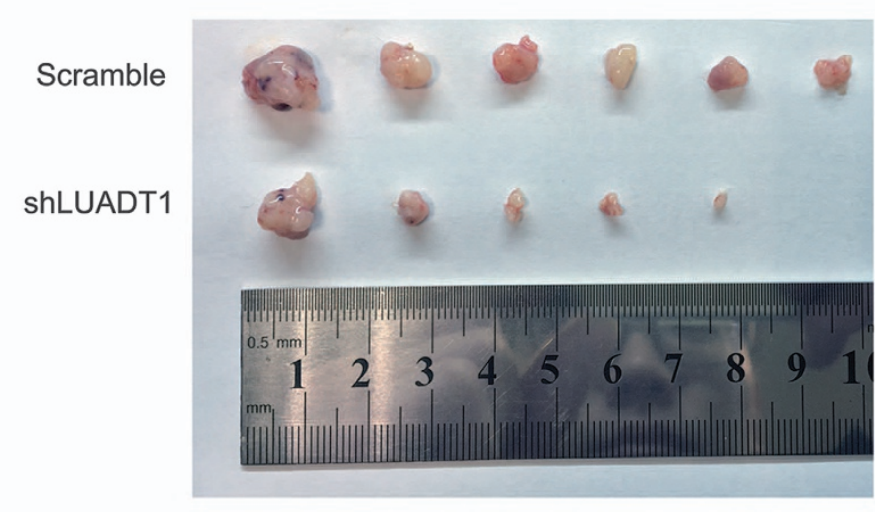

e
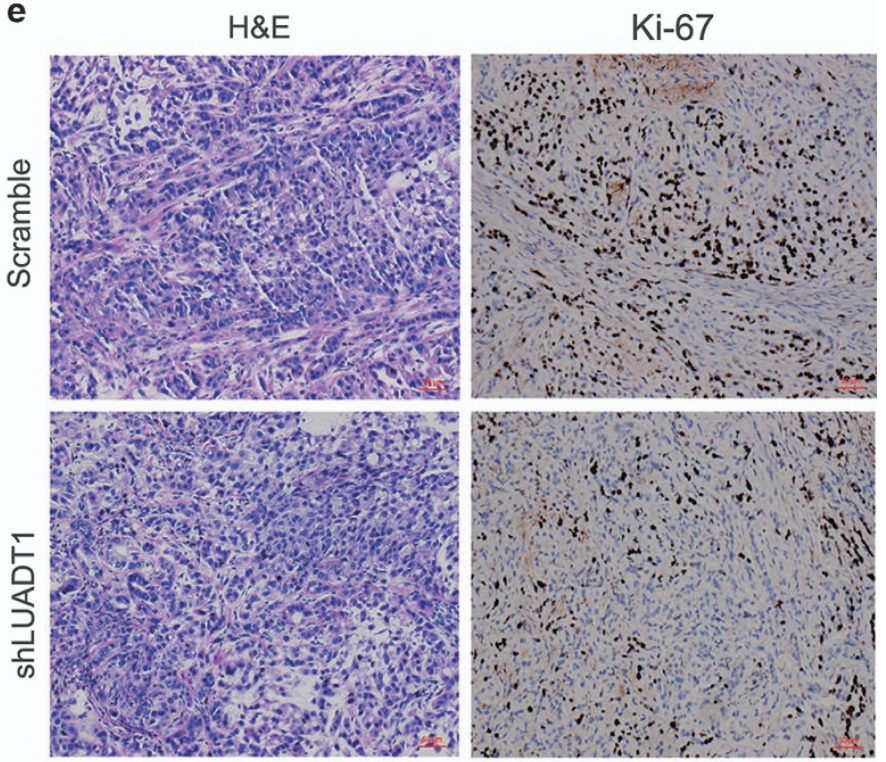

Figure 4 The silencing of LUADT1 inhibited LUAD growth in vivo. LUADT1-targeting or scrambled shRNA was transfected into A549 cells, and then, the cells were injected into nude mice. (a) LUADT1 expression was downregulated in the shLUADT1-transfected cell-derived xenograft tumors. The xenograft tumor weight (c) and volume (b and d) in the shLUADT1 group were significantly lower than those in the scrambled shRNA group. IHC staining was performed on xenograft tumors, and the Ki67 staining signal was weaker in the shLUADT1 group than in the scrambled shRNA group (e). ${ }^{*} P<0.05,{ }^{* \star} P<0.01$. Error bars indicate means \pm S.E.M.

p27 staining was significantly stronger in the xenograft tumors derived from shLUADT1-transfected cells (Figure 6c).

Thus, these lines of evidence demonstrated that LUADT1 binds to SUZ12 and epigenetically inhibits p27 expression by mediating H3K27 trimethylation at the promoter region of p27.

\section{Discussion}

Effective and individualized treatment of LUAD has not been well established. Understanding the gene expression profile and identifying the aberrantly expressed genes in LUAD may represent the crucial nodal points for the diagnosis and the therapeutic intervention of LUAD. Previous studies have demonstrated that IncRNAs have an important role in cancer by functioning as tumor suppressors ${ }^{40,41}$ or oncogenes. ${ }^{10,28,42}$ For lung cancer, several dysregulated IncRNAs have been reported, ${ }^{11,13}$ but these characterized IncRNAs are only the tip of iceberg, as most IncRNAs have not been investigated.

Based on microarray analysis, we determined the expression profiles of IncRNAs and protein-coding genes in five female non-smokers of LUAD and constructed an interaction network between IncRNAs and protein-coding genes, revealing the complex regulatory relationship between different types of genes. By bioinformatics methods, we identified a novel IncRNA, LUADT1. The expression level of LUADT1 was higher in LUAD than LSCC but we failed to find association between LUADT1 expression and smoking (Table 1). Experiments showed that LUADT1 promoted LUAD cell proliferation by epigenetic suppression of p27. Inhibition of LUADT1 significantly inhibited LUAD cell proliferation both in vitro and in vivo, suggesting LUADT1 could be a therapeutic target of LUAD. 
a

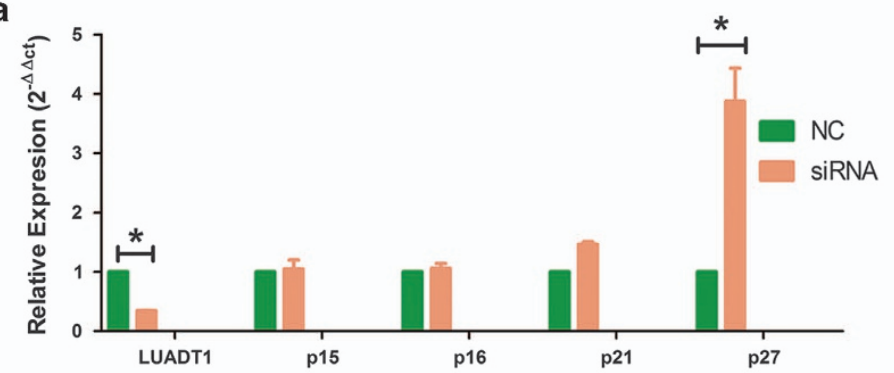

b

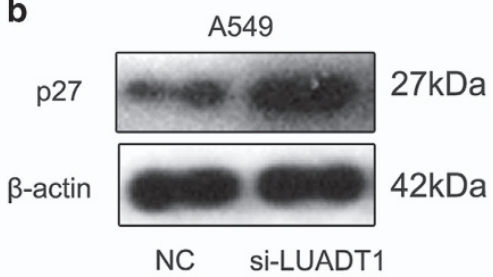

C
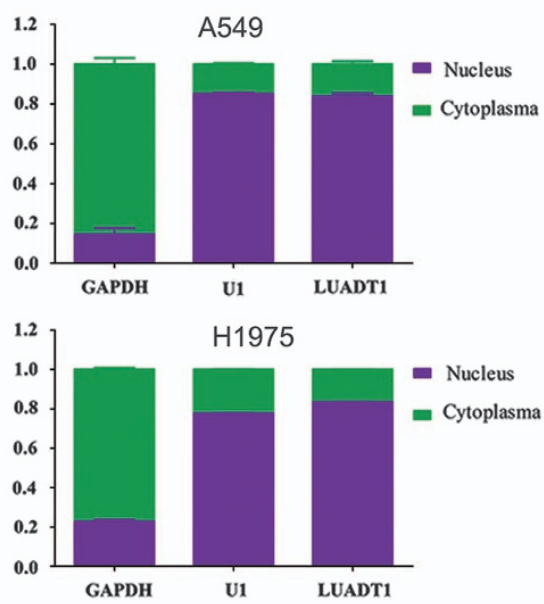

e

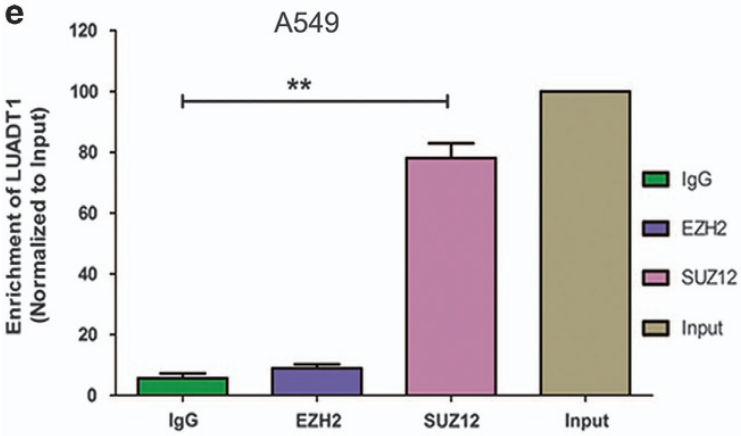

g

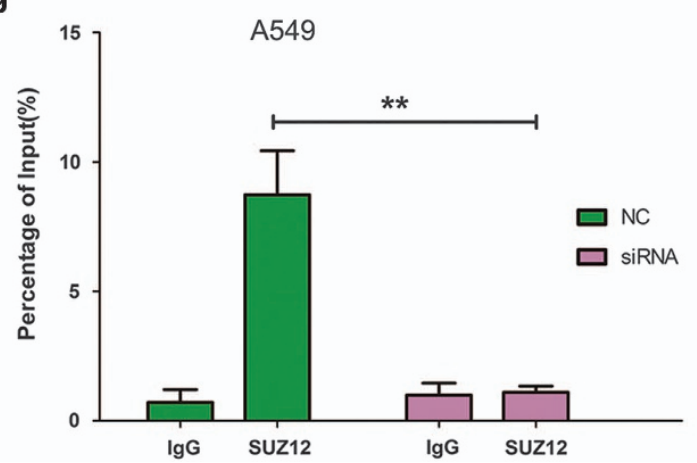

d
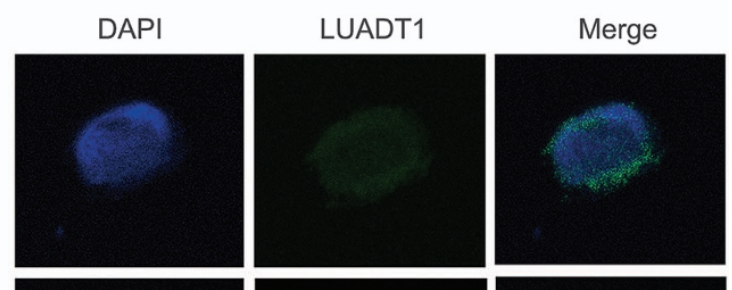

H1975
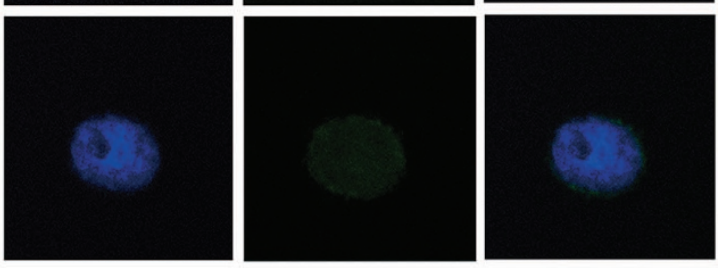$$
\text { f }
$$

A549

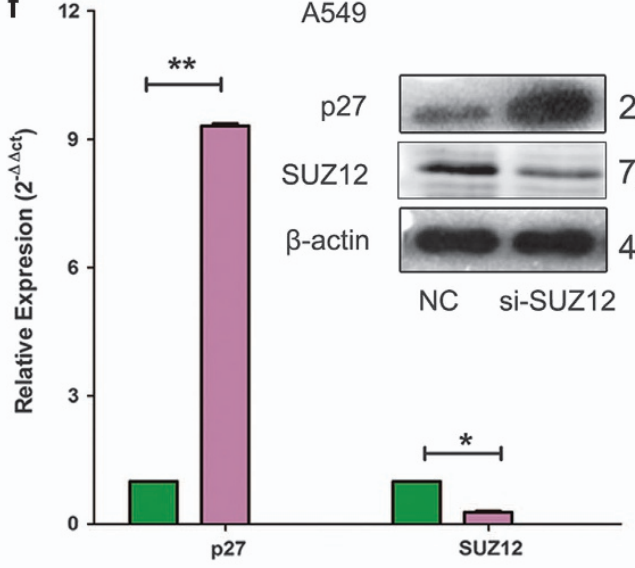

h

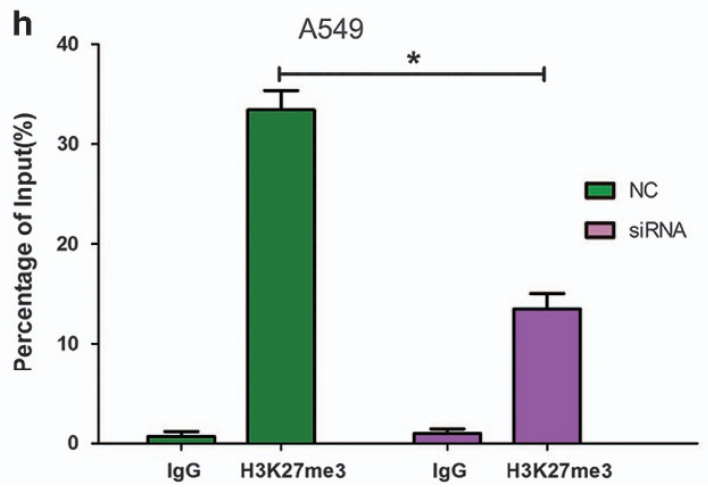

Figure 5 LUADT1 binds to SUZ12 to suppress p27 expression. The expression of cell cycle-related genes was analyzed after LUADT1 knockdown (a). Significant upregulation of p27 was observed and was confirmed by western blot (b). Cell fractionation assay revealed that LUADT1 is predominantly located in nucleus, and GAPDH and small nuclear RNAU1 were used as control genes of cytoplasm and nucleus (c). Fluorescence in situ hybridization assay demonstrated that most LUADT1 was located in nucleus (d). An RIP assay confirmed that LUADT1 binds to SUZ12, although the interaction between EZH2 and LUADT1 was not apparent (e). The silencing of SUZ12 decreased p27 expression at the mRNA and protein levels (f). The enrichment of SUZ12 and trimethylated H3K27 in the promoter region of p27 was detected via ChIP, and this enrichment was decreased after LUADT1 knockdown ( $\mathbf{g}$ and $\mathbf{h}$ ). ${ }^{*} P<0.05,{ }^{\star \star} P<0.01$. Error bars indicate means \pm S.E.M. 

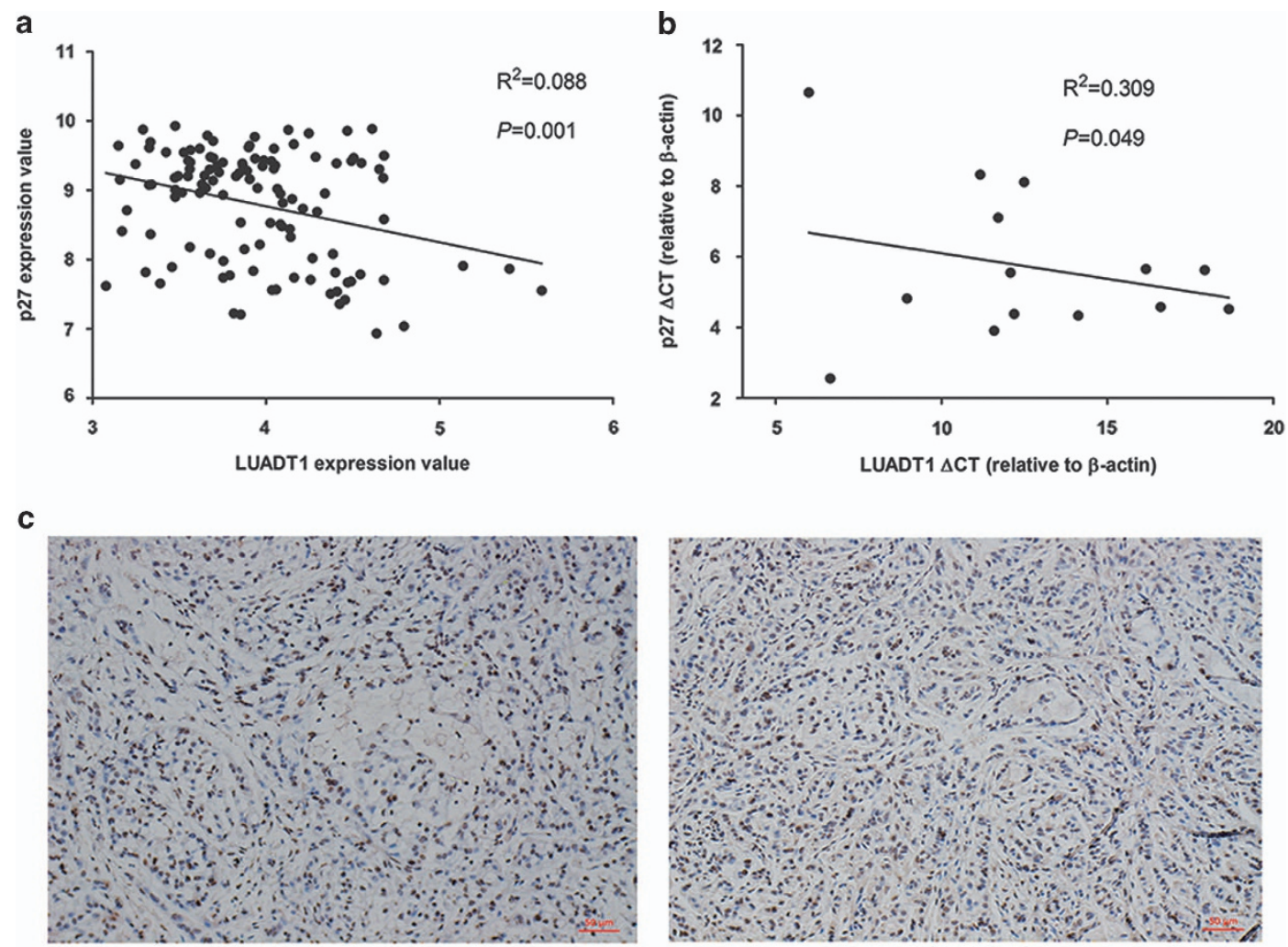

Scramble

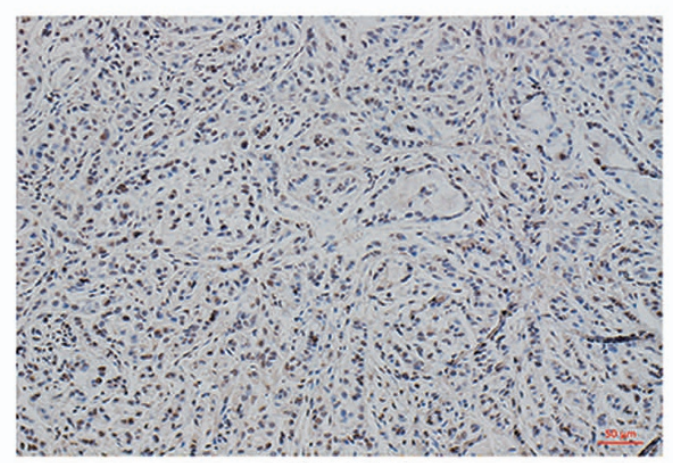

ShLUADT1

Figure 6 Negative correlation between LUADT1 and p27 expression. (a) The LUADT1 and p27 expression levels were negatively correlated in a lung cancer data set of 117 patients (GSE37138). This negative correlation was confirmed in our expression cohort via qRT-PCR (b). IHC staining of xenograft tumors showed that the p27 staining signal was stronger in the shLUADT1-transfected cell-derived xenograft tumors, in which LUADT1 expression was reduced (c)

In most cases, IncRNAs exert their function by binding to various RBPs, such as WDR5, ${ }^{43}$ GADD45A ${ }^{41}$ and hnRNPK. ${ }^{44}$ Among these RBPs, PRC2 has got most attention. PRC2 is a critical regulator of histone modification, which catalyzes the trimethylation of H3K27 to mediate gene silencing. Recent findings implicate that PRC2 is an important driver of tumor development and progression by suppressing various key genes, such as CDH1, DKKI and INK/ARF. ${ }^{45}$ DNA-binding factors are involved the recruitment of PRC2 to specific target genes. Increasing evidence has shown that in addition to proteins, many IncRNAs ${ }^{10,28}$ physically associate with PRC2 and mediate H3K27 trimethylation at distinctive target loci. In this study, we demonstrated that LUADT1 binds to SUZ12 and epigenetically suppresses p27 expression.

p27 is a tumor suppressor that regulates cell cycle proliferation and is often downregulated in cancers. In lung cancer, low p27 expression is associated with poor prognosis. ${ }^{46,47}$ We confirmed that the expression levels of p27 and LUADT1 were negatively correlated in LUAD patients. Moreover, in shLUADT1-transfected cell-derived xenograft tumors, p27 staining was lower than that in control cell-derived xenograft tumors. These data indicated LUADT1 is a robust negative regulator of $\mathrm{p} 27$. We also found that LUADT1 expression significantly positively correlated with $T$ stage (Figure 2h). This result is consistent with the function of LUADT1 because LUADT1 may promote proliferation of
LUAD; thus, a higher expression level of LUADT1 indicates a larger tumor size, that is, $T$ stage.

Owing to the prognostic value of p27, the negative correlation between p27 and LUADT1 implies that LUADT1 may also be a prognostic marker for LUAD. However, owing to the limited follow-up information, the prognostic performance of LUADT1 has not been validated. It is highly possible that LUADT1 could regulate a set of other genes and RNA-sequencing or microarray analysis following LUADT1 knockdown may help to identify the downstream targets of LUADT1.

In this study, we identified a set of aberrantly expressed IncRNAs and protein-coding genes in LUAD. Our study paves the road for future investigations of biomarkers for LUAD and the comprehensive understanding of the molecular mechanisms by which IncRNAs affect LUAD.

\section{Materials and Methods}

Patients and tissue samples. This study was approved by the Ethics Committee of Cancer Institute of Jiangsu Province. Paired non-small cell lung cancer tissues and adjacent non-tumor tissues were obtained from patients who received surgical resection of NSCLC between 2012 and 2014 at the Department of Thoracic Surgery of Cancer Institute of Jiangsu Province, China. The pathological stage, grade and nodal status of all paired tumor and non-tumor tissues were confirmed by experienced pathologists. Clinical and pathological characteristics were also collected for each patient. Informed written consent was obtained from all patients included in this study. 
Microarray and computational analysis. The microarray experiment was performed by CapitalBio Corporation, Beijing, China. Expression profiling of IncRNAs and protein-coding genes was performed using the Agilent human IncRNA +mRNA array V.2.0 platform (Agilent Technologies, Santa Clara, CA, USA). The microarray data have been submitted to the Gene Expression Omnibus and the data could be accessed by the accession number, GSE66654. Five LUAD tissues corresponding non-tumor tissues (Supplementary Table 1) were used for microarray analysis. For TF-gene network, the sequences of differentially expressed genes were retrieved and analyzed with the Jemboss software (The European Molecular Biology Open Software Suite Team) to identify the relationships between genes and TFs. Next, a transcription TF-gene network was constructed based on the interactions between genes and TFs. We built IncRNA-protein-coding genes network to identify the interactions between protein-coding genes and IncRNA. ${ }^{48}$ For each gene-IncRNA, gene-gene or IncRNA-IncRNA pair, we calculated the Pearson correlation coefficient and selected the significantly correlated pairs to construct the network. ${ }^{23}$

Cell lines and culture conditions. A549, H1975, H358, H1650 and H1299 cells were cultured in RPMI 1640 medium (KeyGEN, Nanjing, China) and Pc9 and HBE cells were cultured in DMEM medium, supplemented with $10 \% \mathrm{FBS}$ (GIBCO-BRL, Invitrogen, Carlsbad, CA, USA), $100 \mathrm{U} / \mathrm{ml}$ penicillin and $100 \mathrm{mg} / \mathrm{ml}$ streptomycin (KeyGEN) in humidified air at $37^{\circ} \mathrm{C}$ with $5 \% \mathrm{CO}_{2}$.

RNA extraction and qRT-PCR. The total RNA was extracted from tissues or cultured cells with TRIzol reagent (Invitrogen, Grand Island, NY, USA), according to the manufacturer's protocol. One microgram total RNA was reverse transcribed in a final volume of $20 \mu \mathrm{l}$ using PrimerScript RT Master Mix (Takara, Dalian, China; cat. no. RR036A). qRT-PCR was performed as previously described. ${ }^{22}$

Subcellular fractionation location. The separation of the nuclear and cytosolic fractions was performed using the PARIS Kit (Life Technologies, Carlsbad, CA, USA) according to the manufacturer's instructions.

Fluorescence in situ hybridization. Cells were fixed in $4 \%$ formaldehyde/ $5 \%$ acetic acid for $15 \mathrm{~min}$ followed by washes with PBS. The fixed cells were further treated with pepsin $(1 \%$ in $10 \mathrm{mM} \mathrm{HCl})$ and subsequent dehydration through $70 \%$, $90 \%$ and $100 \%$ ethanol. The air-dried cells were subjected to incubation with $40 \mathrm{nM}$ FISH probe in hybridization buffer $(100 \mathrm{mg} / \mathrm{ml}$ dextran sulfate, $10 \%$ formamide in $2 \times \mathrm{SSC}$ ) at $80^{\circ} \mathrm{C}$ for $2 \mathrm{~min}$. The hybridization was performed at $55^{\circ} \mathrm{C}$ for $2 \mathrm{~h}$ and the slide was washed with $0.1 \times$ SSC at $65^{\circ} \mathrm{C}$ followed by dehydration through $70 \%$, $90 \%$ and $100 \%$ ethanol. The air-dried slide was mounted with Prolong Gold Antifade Reagent with DAPI for detection. RNA FISH probe were designed and synthesized by Bogu Co., Ltd (Shanghai, China). Probe sequences are listed in Supplementary Table 2.

Transfection of cell lines. Typically, LUAD cells were seeded at six-well plates and then transfected in the next day with specific siRNA $(100 \mathrm{nM})$ or control siRNA (100 nM) using Lipofectamine RNAi MAX, according to the manufacturer's protocol (Invitrogen). The shRNA sequence of LUADT1 (5'-GATCCCCAGC AATCCTCTTACAGCAATTCAAGAGATTGCTGTAAGAGGATTGCTTTTTTTGGAAA-3') was cloned into $\mathrm{pENTR/U6}$ vector. The primer sequences and siRNA sequences are summarized in Supplementary Table 2.

Cell proliferation assay. A549 and $\mathrm{H} 1975$ cells were harvested $24 \mathrm{~h}$ post transfection by trypsinization. The Cell Counting Kit-8 assay was used to determine cell growth according to the manufacturer's instructions (KeyGEN). BrdU experiments were performed using a BrdU Cell Proliferation Assay Kit (Millipore, Billerica, MA, USA; cat. no. 2750) according to the manufacturer's instructions. The higher $\mathrm{OD}$ reading represents the higher BrdU concentration in the sample. The absorbance was measured at $450 \mathrm{~nm}$ with an ELx-800 Universal Microplate Reader (BioTek, Winooski, VT, USA). Each experiment was repeated at least three times independently.

Flow cytometric analysis. For cell cycle analysis, cells were cultured with serum-free medium $24 \mathrm{~h}$ before transfection to induce cell cycle synchronization. Transfected cells were harvested after transfection by trypsinization. After the double staining with fluorescein isothiocyanate (FITC)-Annexin V and propidium iodide was done by the FITC Annexin V Apoptosis Detection Kit (BD Biosciences, San Jose, CA, USA) according to the manufacturer's recommendations. The cells were analyzed with a flow cytometry (FACScan; BD Biosciences) equipped with a Cell Quest software (BD Biosciences). Cells for cell cycle analysis were stained with propidium oxide by the Cycle TEST PLUS DNA Reagent Kit (BD Biosciences) following the protocol and analyzed by FACScan. The percentage of the cells in G0G1, S and G2-M phase were counted and compared.

Western blot assay. Cells were harvested, and protein was extracted from transfected cells and quantified as previously described ${ }^{49}$ using $12 \%$ or $4-20 \%$ polyacrylamide gradient SDS gel. Anti- $\beta$-actin and anti-SUZ12 were from Abcam (Hong Kong, China). Anti-p27, anti-cyclin D1, anti-CDK4 and anti-CDK6 were from Cell Signaling Technology (Boston, MA, USA).

RNA immunoprecipitation. RIP experiments were performed using a Magna RIP RNA-Binding Protein Immunoprecipitation Kit (Millipore) according to the manufacturer's instructions. Antibodies of EZH2 and SUZ12 were from Abcam.

ChIP assays. The ChIP assays were performed using EZ-CHIP KIT according to the manufacturer's instruction (Millipore). H3K27 antibody was from Millipore. The ChIP primer sequences were provided in Supplementary Table S2.

Immunohistochemistry. Xenograft tumor tissue samples were immunostained for p27 and Ki67. Anti-Ki67 was from Santa Cruz Biotechnology (Dallas, TX, USA).

Xenograft experiment. Transient transfection was performed in A549 cells with shLUADT1 or scrambled control sequence using Lipofectamine 2000 (Invitrogen). After $48 \mathrm{~h}$ of transfection, the cells were collected and injected into either side of the posterior flank of the same male BALB/c nude mouse. The tumor volumes and weights were measured every 2 days in the mice; the tumor volumes were measured as length $\times$ width $^{2} \times 0.5$. Sixteen days after injection, the mice were killed, the tumor weights were measured and the tumors were collected for further analysis. The LUADT1 levels were determined by qRT-PCR.

Statistical analysis. Student's t-test, one-way ANOVA analysis and Spearman test were performed to analyze the data using SPSS 18.0 software (Armonk, NY, USA). $P<0.05$ was considered statistically significant.

\section{Conflict of Interest}

The authors declare no conflict of interest.

Acknowledgements. This study is founded by the Natural Science Foundation of China (81372321 to Lin Xu; 81201830 and 81472200 to Rong Yin), Natural Science Foundation for High Education of Jiangsu Province (13KJB320010 to Rong Yin), Jiangsu Provincial Special Program of Medical Science (BL2012030 to Lin Xu) and Jiangsu province ordinary university graduate student research innovation project for 2013 (CXLX13_571 to Mantang Qiu).

1. Jemal A, Bray F, Center MM, Ferlay J, Ward E, Forman D. Global cancer statistics. $C A$ Cancer J Clin 2011; 61: 69-90.

2. Couraud S, Zalcman G, Milleron B, Morin F, Souquet PJ. Lung cancer in never smokersa review. Eur J Cancer 2012; 48: 1299-1311.

3. Beasley MB, Brambilla E, Travis WD. The 2004 World Health Organization classification of lung tumors. Semin Roentgenol 2005; 40: 90-97.

4. Govindan R, Ding L, Griffith M, Subramanian J, Dees ND, Kanchi KL et al. Genomic landscape of non-small cell lung cancer in smokers and never-smokers. Cell 2012; 150: 1121-1134.

5. Ponting CP, Oliver PL, Reik W. Evolution and functions of long noncoding RNAs. Cell 2009; 136: 629-641.

6. Mercer TR, Dinger ME, Mattick JS. Long non-coding RNAs: insights into functions. Nat Rev Genet 2009; 10: 155-159.

7. Wang $P, X u e Y$, Han $Y$, Lin L, Wu C, Xu S et al. The STAT3-binding long noncoding RNA IncDC controls human dendritic cell differentiation. Science 2014; 344: 310-313.

8. Kretz M, Siprashvili Z, Chu C, Webster DE, Zehnder A, Qu K et al. Control of somatic tissue differentiation by the long non-coding RNA TINCR. Nature 2013; 493: 231-235.

9. Hung CL, Wang LY, Yu YL, Chen HW, Srivastava S, Petrovics G et al. A long noncoding RNA connects c-Myc to tumor metabolism. Proc Natl Acad Sci USA 2014; 111: 18697-18702.

10. Gupta RA, Shah N, Wang KC, Kim J, Horlings HM, Wong DJ et al. Long non-coding RNA HOTAIR reprograms chromatin state to promote cancer metastasis. Nature 2010; 464: 1071-1076. 
11. Yang F, Huo XS, Yuan SX, Zhang L, Zhou WP, Wang F et al. Repression of the long noncoding RNA-LET by histone deacetylase 3 contributes to hypoxia-mediated metastasis. Mol Cell 2013; 49: 1083-1096.

12. Xing Z, Lin A, Li C, Liang K, Wang S, Liu $Y$ et al. IncRNA directs cooperative epigenetic regulation downstream of chemokine signals. Cell 2014; 159: 1110-1125.

13. Ji P, Diederichs S, Wang W, Boing S, Metzger R, Schneider PM et al. MALAT-1, a nove noncoding RNA, and thymosin beta4 predict metastasis and survival in early-stage nonsmall cell lung cancer. Oncogene 2003; 22: 8031-8041.

14. Yang Z, Zhou L, Wu LM, Lai MC, Xie HY, Zhang F et al. Overexpression of long non-coding RNA HOTAIR predicts tumor recurrence in hepatocellular carcinoma patients following liver transplantation. Ann Surg Oncol 2011; 18: 1243-1250.

15. Schmidt LH, Spieker T, Koschmieder S, Schaffers S, Humberg J, Jungen D et al. The long noncoding MALAT-1 RNA indicates a poor prognosis in non-small cell lung cancer and induces migration and tumor growth. J Thorac Oncol 2011; 6: 1984-1992.

16. Wang L, Zhao Y, Bao X, Zhu X, Kwok YK, Sun K et al. LncRNA Dum interacts with Dnmts to regulate Dppa2 expression during myogenic differentiation and muscle regeneration. Cell Res 2015; 25: 335-350.

17. Ranzani V, Rossetti G, Panzeri I, Arrigoni A, Bonnal RJ, Curti S et al. The long intergenic noncoding RNA landscape of human lymphocytes highlights the regulation of $T$ cell differentiation by linc-MAF-4. Nat Immunol 2015; 16: 318-325.

18. Kornfeld JW, Bruning JC. Regulation of metabolism by long, non-coding RNAs. Front Genet 2014; 5 : 57.

19. Kim T, Jeon YJ, Cui R, Lee JH, Peng Y, Kim SH et al. Role of MYC-regulated long noncoding RNAs in cell cycle regulation and tumorigenesis. J Natl Cancer Inst 2015; e-pub ahead o print 6 February 2015; doi:10.1093/jnci/dju505.

20. Uchida S, Dimmeler S. Long noncoding RNAs in cardiovascular diseases. Circ Res 2015; 116: $737-750$.

21. Tsai MC, Spitale RC, Chang HY. Long intergenic noncoding RNAs: new links in cancer progression. Cancer Res 2011; 71: 3-7.

22. Qiu MT, Xu YT, Yang X, Wang J, Hu JW, Xu L et al. CCAT2 is a lung adenocarcinomaspecific long non-coding RNA and promotes invasion of non-small cell lung cancer. Tumour Biol 2014; 35: 5375-5380.

23. Prieto C, Risueno A, Fontanillo C, De las Rivas J. Human gene coexpression landscape: confident network derived from tissue transcriptomic profiles. PLoS One 2008; 3: e3911.

24. Vermeirssen V, Barrasa MI, Hidalgo CA, Babon JA, Sequerra R, Doucette-Stamm $L$ et al. Transcription factor modularity in a gene-centered $C$. elegans core neuronal protein-DNA interaction network. Genome Res 2007; 17: 1061-1071.

25. Basak S, Ramesh AM, Kesari V, Parida A, Mitra S, Rangan L. Genetic diversity and relationship of Hedychium from Northeast India as dissected using PCA analysis and hierarchical clustering. Meta Gene 2014; 2: 459-468.

26. Cameron DA, Middleton FA, Chenn A, Olson EC. Hierarchical clustering of gene expression patterns in the Eomes+lineage of excitatory neurons during early neocortical development. BMC Neurosci 2012; 13: 90

27. Nayak RR, Kearns M, Spielman RS, Cheung VG. Coexpression network based on natura variation in human gene expression reveals gene interactions and functions. Genome Res 2009; 19: 1953-1962.

28. Yang $\mathrm{F}$, Zhang $\mathrm{L}$, Huo XS, Yuan JH, Xu D, Yuan SX et al. Long noncoding RNA high expression in hepatocellular carcinoma facilitates tumor growth through enhancer of zeste homolog 2 in humans. Hepatology 2011; 54: 1679-1689.

29. Smith LT, Lin M, Brena RM, Lang JC, Schuller DE, Otterson GA et al. Epigenetic regulation of the tumor suppressor gene TCF21 on 6q23-q24 in lung and head and neck cancer. Proc Nat Acad Sci USA 2006; 103: 982-987.

30. Tessema M, Willink R, Do K, Yu YY, Yu W, Machida EO et al. Promoter methylation of genes in and around the candidate lung cancer susceptibility locus 6q23-25. Cancer Res 2008; 68 1707-1714.

31. Lin MF, Jungreis I, Kellis M. PhyloCSF: a comparative genomics method to distinguish protein coding and non-coding regions. Bioinformatics 2011; 27: i275-i282.
32. Wang L, Park HJ, Dasari S, Wang S, Kocher JP, Li W, CPAT. Coding-potential assessment tool using an alignment-free logistic regression model. Nucleic Acids Res 2013; 41: e74.

33. Pathmanathan N, Balleine RL. Ki67 and proliferation in breast cancer. J Clin Pathol 2013; 66: 512-516.

34. Yoon MK, Mitrea DM, Ou L, Kriwacki RW. Cell cycle regulation by the intrinsically disordered proteins p21 and p27. Biochem Soc Trans 2012; 40: 981-988.

35. Khalil AM, Guttman M, Huarte M, Garber M, Raj A, Rivea Morales D et al. Many human large intergenic noncoding RNAs associate with chromatin-modifying complexes and affect gene expression. Proc Natl Acad Sci USA 2009; 106: 11667-11672.

36. Muppirala UK, Honavar VG, Dobbs D. Predicting RNA-protein interactions using only sequence information. BMC Bioinformatics 2011; 12: 489.

37. Agostini F, Zanzoni A, Klus P, Marchese D, Cirillo D, Tartaglia GG. catRAPID omics: a web server for large-scale prediction of protein-RNA interactions. Bioinformatics 2013; 29 : 2928-2930.

38. Brockdorff N. Noncoding RNA and polycomb recruitment. RNA 2013; 19: 429-442.

39. Baty F, Rothschild S, Fruh M, Betticher D, Droge C, Cathomas R et al. EGFR exon-level biomarkers of the response to bevacizumab/erlotinib in non-small cell lung cancer. PLoS One 2013; 8: e72966.

40. Yu W, Qiao Y, Tang X, Ma L, Wang Y, Zhang X et al. Tumor suppressor long non-coding RNA, MT1DP is negatively regulated by YAP and Runx2 to inhibit FoxA1 in liver cancer cells. Cell Signal 2014; 26: 2961-2968.

41. Arab K, Park YJ, Lindroth AM, Schafer A, Oakes C, Weichenhan D et al. Long noncoding RNA TARID directs demethylation and activation of the tumor suppressor TCF21 via GADD45A. Mol Cell 2014; 55: 604-614.

42. Redis RS, Sieuwerts AM, Look MP, Tudoran O, Ivan C, Spizzo R et al. CCAT2, a novel long non-coding RNA in breast cancer: expression study and clinical correlations. Oncotarget 2013; 4: 1748-1762.

43. Yang YW, Flynn RA, Chen Y, Qu K, Wan B, Wang KC et al. Essential role of IncRNA binding for WDR5 maintenance of active chromatin and embryonic stem cell pluripotency. Elife 2014; 3: e02046.

44. Huarte M, Guttman M, Feldser D, Garber M, Koziol MJ, Kenzelmann-Broz D et al. A large intergenic noncoding RNA induced by p53 mediates global gene repression in the p53 response. Cell 2010; 142: 409-419.

45. Chang CJ, Hung MC. The role of EZH2 in tumour progression. Br J Cancer 2012; 106: 243-247.

46. Singhal S, Vachani A, Antin-Ozerkis D, Kaiser LR, Albelda SM. Prognostic implications of cell cycle, apoptosis, and angiogenesis biomarkers in non-small cell lung cancer: a review. Clin Cancer Res 2005; 11: 3974-3986.

47. Shen J, Yin JY, Li XP, Liu ZQ, Wang Y, Chen J et al. The prognostic value of altered elF3a and its association with p27 in non-small cell lung cancers. PLoS One 2014; 9: e96008.

48. Pujana MA, Han JD, Starita LM, Stevens KN, Tewari M, Ahn JS et al. Network modeling links breast cancer susceptibility and centrosome dysfunction. Nat Genet 2007; 39: 1338-1349.

49. Yang X, Zhang Z, Qiu MT, Hu JW, Fan XH, Wang J et al. Glypican-5 is a novel metastasis suppressor gene in non-small cell lung cancer. Cancer Lett 2013; 341: 265-273.

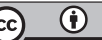

Cell Death and Disease is an open-access journal published by Nature Publishing Group. This work is licensed under a Creative Commons Attribution 4.0 International License. The images or other third party material in this article are included in the article's Creative Commons license, unless indicated otherwise in the credit line; if the material is not included under the Creative Commons license, users will need to obtain permission from the license holder to reproduce the material. To view a copy of this license, visit http://creativecommons.org/licenses/by/4.0/ 